\title{
DROPS IN PHYSICAL PERFORMANCE DURING INTERMITTENT SMALL-SIDED AND CONDITIONED GAMES IN PROFESSIONAL SOCCER PLAYERS
}

original paper

( ) University School of Physical Education in Wroclaw

DOI: https://doi.org/10.5114/hm.2020.88148

\author{
FILIPE MANUEL CLEMENTE ${ }^{1,2}$, ALIREZA RABBANI ${ }^{3}$, RUBEN FERREIRA $^{3,4}$, \\ JOÃO PEDRO ARAÚJOO \\ ${ }^{1}$ Polytechnic Institute of Viana do Castelo, School of Sport and Leisure, Melgaço, Portugal \\ ${ }^{2}$ Instituto de Telecomunicações, Delegação da Covilhã, Portugal \\ ${ }^{3}$ Medical and Performance Department, Sporting Clube de Portugal, Lisbon, Portugal \\ ${ }^{4}$ Football Medicine ${ }^{\circledR}$, Portugal
}

\section{ABSTRACT}

Purpose. The purpose of this study was to identify variations in external load measures between sets (3 $\times 4$ '/2' rest) of small-sided and conditioned game formats (goalkeeper +5 vs. $5+$ goalkeeper with specific tactical demands).

Methods. Overall, 10 male professional soccer players (age: $28.1 \pm 3.8$ years, experience: $7.9 \pm 2.9$ years, height: $180.3 \pm$ $5.8 \mathrm{~cm}$, weight: $76.5 \pm 6.3 \mathrm{~kg}$ ) from a team competing in the first Portuguese league and in the UEFA Europa league participated in the study. Distance covered, high intensity running (HIR), maximal velocity (MV), high speed running (HSR), very high intensity running (VHIR), and player load (PL) were monitored by using global positioning system units.

Results. Very large decreases in HIR (-62.6, effect size [ES]: -2.04) were found from set 1 to set 3. Large decreases in distance covered (-12.8\%, ES: -1.28$)$ and MV (-19.4, ES: -1.95$)$ were observed from the first to the third set. Large decreases in HSR (-80.2\%, ES: -1.78$)$, moderate decreases in PL (-7.6\%, ES: -0.64$)$, and large decreases in VHIR (-83.0\%, ES: -1.88$)$ were reported between the first and the third set.

Conclusions. The results of the present study indicate a significant drop in external load performance when comparing the first and the second sets with the third set.

Key words: association football, drill-based tasks, sided-games, training load, external load

\section{Introduction}

Small-sided games (SSGs) are smaller and adjusted versions of the formal soccer game format which are implemented into training programs to increase the individual participation of players, augment the perception of players for specific tactical principles, increase players' acute physiological responses, and impose highly demanding physical stimuli [1, 2]. Usually, these games can be classified into the following categories on the basis of the number of players involved and the format: extreme ( 1 vs. 1$)$, small ( 2 vs. 2 to 4 vs. 4 ), medium ( 5 vs. 5 to 8 vs. 8), and large (9 vs. 9 to 11 vs. 11) [3]. Depending on the format, the physiological stimuli and the physical demands vary, with high acute physiological responses [4-6] and high mechanical load [7] being reported consistently during extreme and small formats. The effects of SSGs on physiological and physical demands also depend on other concurrent task conditions, such as the size of the pitch (and the relative individual playing area), rules (e.g., limitations on touches, presence or absence of goals, space limitations, type of marking), coaches' encouragement [8], inclusion of goalkeepers (GK) [9], or training regimen (e.g., continuous vs. intermittent) $[1,10,11]$.

The use of different training regimens may lead to variations in physiological and physical stimuli [12], considering that these games are usually used to work on the players' anaerobic thresholds under conditions that contribute to declines in performance $[13,14]$. For that reason, some studies have aimed to identify variations in load measures within sessions and between sets $[15,16]$.

Correspondence address: Filipe Manuel Clemente, Complexo Desportivo e Lazer de Melgaço, Monte de Prado, 4960-320, Melgaço, Portugal, e-mail: filipe.clemente5@gmail.com

Received: January 11, 2019

Accepted for publication: May 31, 2019

Citation: Clemente FM, Rabbani A, Ferreira R, Araújo JP. Drops in physical performance during intermittent small-sided and conditioned games in professional soccer players. Hum Mov. 2020;21(1):7-14; https://doi.org/10.5114/hm.2020.88148. 
F. Clemente, A. Rabbani, R. Ferreira, J.P. Araújo, Performance dropping in small-sided games

In the context of the small format (3 vs. 3) played by amateur and professional players, variations in heart rate, perceived effort, and technical actions were tested between long sets $(3 \times 6$ minutes $/ 2$ minutes rest), medium sets $(3 \times 4$ minutes $/ 2$ minutes rest), and short sets $(3 \times 2$ minutes/2 minutes rest) [15]. In that study, it was found that long sets contributed to decreases in heart rate in comparison with medium and short sets. However, no significant differences were found in terms of perceived effort or technical actions [15]. Using a similar experimental design with different SSG formats ( 2 vs. 2 , 3 vs. 3 , and 4 vs. 4 ) applied in under- 17 players, it was observed that short sets $(6 \times 2$ minutes $)$ elicited significantly lower heart rate responses and blood lactate concentrations in comparison with long $(2 \times 6$ minutes $)$ and medium $(3 \times 4$ minutes $)$ sets; the short sets also contributed to shorter distances covered in low-intensity activities and significant increases in moderate- and high-intensity activities [16].

By identifying the effects of different SSG regimens on the load variables objectively measured during training sessions, coaches can determine the most appropriate combination of times for sets and rest periods to avoid significant within-session drops. Although a massive number of studies have been conducted on SSGs [1, 11, 17], few of them, as far as we know, have observed between-set variations and their effects on external load measures. In fact, the studies reporting evidence about inter-set variations only reported internal load variables $[5,16]$ and for that reason it is important to add new evidence about the external load variations aiming to provide useful information for coaches and new insights for the scientific community. Moreover, comparisons between intermittent regimens $[5,16]$ are usually conducted by using simple formats without considering the concurrent task conditions that are very common in real training scenarios. For those reasons, and with the aim of filling this gap in the SSG literature, this study analyses variations of external load measures between sets of medium-sided and conditioned games played by elite and professional soccer players. We hypothesized that inter-set variations of external load variables would occur, namely decreasing the values with the progression of the sets. We assumed that possibly the largest decreases would occur when comparing the first with the last set.

\section{Material and methods}

\section{Participants}

A total of 10 elite and professional male soccer players (age: $28.1 \pm 3.8$ years, experience at professional competitive level: $7.9 \pm 2.9$ years, height: $180.3 \pm 5.8$ $\mathrm{cm}$, weight: $76.5 \pm 6.3 \mathrm{~kg}$ ) from a team competing in the first Portuguese league and in the UEFA Europa league participated in this study. The sample included 4 defenders, 4 midfielders, and 2 forwards, equally distributed in 2 teams. The players usually trained 6 times a week, with an average training session time of 70 minutes. The participants were informed of the experimental design and were made aware that the training load monitoring process would take place during the drills as part of their daily practice. They were also informed of the potential implications, risks, and benefits of the study.

\section{Experimental approach}

A cross-sectional design study was conducted to compare within-session variations in distance covered, high intensity running (HIR), maximal velocity (MV), high speed running (HSR), very high intensity running (VHIR), and player load (PL) during a constrained GK + 5 vs. 5 + GK intermittent SSG.

The study was conducted in the middle of the season (11 weeks after the beginning of the pre-season). The day on which data were collected was preceded by 48 hours of rest, 72 hours after the last high load session and 144 hours after the last match. The players were distributed to teams on the basis of their playing position by the head coach, who considered homogenization of the competitive level of players when doing so. The distribution of the players did not change during the SSG sets. Each player was monitored by an individual global positioning system (GPS) unit. The SSGs were played on natural turf at 11:00 a.m. at an average temperature of $13^{\circ} \mathrm{C}$ and a relative humidity of $55 \%$. The SSGs were played without rainy weather conditions. They were preceded by a warm-up protocol consisting of jogging, lower-limb dynamic stretching, and mobility, agility, and speed drills. The SSG were the main exercise of the training, thus ensuring the maximal exertion and commitment level of the players.

\section{Small-sided game}

The GK + 5 vs. 5 + GK format played with official goals was implemented with the following task con- 
ditions: (i) the player had to be in the opponent's half of the pitch when scoring for the goal to be counted; (ii) offside rules applied; and (iii) there was a 2-touch limitation. The size of the pitch was $60 \times 30 \mathrm{~m}\left(1800 \mathrm{~m}^{2}\right)$. Thus, the individual playing area per player was $150 \mathrm{~m}^{2}$. The training regimen consisted of 3 sets of 4 minutes with 2 minutes of rest between sets. Each team was composed of 2 defenders, 2 midfielders, and 1 forward, who had similar skills and fitness levels as determined by preliminary assessments. No strategic or tactical instructions were provided to players during the matches. Only verbal encouragement was given to ensure the high commitment level of players. Six balls were placed around the pitch to reduce the time needed for ball repositioning if the ball in play went out of bounds. Excluding the specific SSG task conditions, all rules followed official soccer rules.

\section{Global positioning system}

Each player wore a vest with a geolocation tracker operating at $10 \mathrm{~Hz}$ (GPS, OptimeEye S5, Catapult, Australia) equipped with an inertial measurement unit (100 Hz, 3 axes). The validity and reliability of the device can be found in previous studies [18]. The device was placed in a bag on the vest located at the dorsal region of the players. Immediately after the end of the session, the data were exported and treated on the proper software (OpenField 1.21.1, Catapult, Australia). The following measures were obtained and standardized per minute: (a) distance covered; (b) HIR, consisting of distance covered above a speed of $14.4 \mathrm{~km} / \mathrm{h}$; (c) MV, consisting of the peak velocity of each set; (d) HSR, consisting of distance covered at speeds between 19.8 and $25.1 \mathrm{~km} / \mathrm{h}$; (e) VHIR, consisting of distance covered at speeds above $19.8 \mathrm{~km} / \mathrm{h}$; and (f) $\mathrm{PL}$, consisting of the sum of instantaneous accelerations in the 3 axes (anteroposterior, mediolateral, and craniocaudal).

\section{Statistical procedures}

The results are presented as either means and standard deviations $(S D)$ or percentage of differences and $90 \%$ confidence interval $(90 \% \mathrm{CI})$. The normality of the sample was preliminarily tested. Between-SSG-set differences were analysed by using the standardized differences of the effect size (ES), with a 90\% CI [19]. The following thresholds were used to classify the magnitude of inferences: $<0.2$, trivial; 0.2-0.6, small; 0.6-1.2, moderate; $1.2-2.0$, large; > 2.0, very large [20]. Probabilities were calculated considering the smallest worthwhile changes (SWC, $0.2 \times$ between-subject $S D$ ) [21]. The scale for qualitative probabilities was assumed as follows: $25-75 \%$, possible; 75-95\%, likely; 95-99\%, very likely; > 99\%, almost certain [21].

\section{Ethical approval}

The research related to human use has complied with all the relevant national regulations and institutional policies, has followed the tenets of the Declaration of Helsinki, and has been approved by the authors' institutional review board or an equivalent committee.

\section{Informed consent}

Informed consent has been obtained from all individuals included in this study.

\section{Results}

Comparisons of external load variables were made between sets, and the descriptive statistics can be observed in Table 1. The highest averages of distance covered $(122.36 \mathrm{~m} / \mathrm{min})$, HIR $(27.03 \mathrm{~m} / \mathrm{min})$, MV (24.84 km/h), HSR (5.59 m/min), VHIR $(6.97 \mathrm{~m} / \mathrm{min})$, and PL (12.68 U.A./min) were found during the second set. On the other hand, the lowest values of all external load variables were observed in the third set. The HSR

Table 1. Descriptive statistics of external load variables during the 3 sets of conditioned $6 \times 6$ format

\begin{tabular}{|c|c|c|c|c|}
\hline & $\begin{array}{c}\text { Set } 1 \\
\text { Mean }(S D)\end{array}$ & $\begin{array}{c}\text { Set } 2 \\
\text { Mean }(S D)\end{array}$ & $\begin{array}{c}\text { Set } 3 \\
\text { Mean }(S D)\end{array}$ & $C V(\%)$ \\
\hline Distance $(\mathrm{m} / \mathrm{min})$ & $119.13(11.43)$ & $122.36(7.44)$ & $103.75(8.83)$ & 8.64 \\
\hline HIR (m/min) & $23.70(8.72)$ & $27.03(10.92)$ & $9.61(4.89)$ & 45.96 \\
\hline MV (km/h) & $24.43(2.35)$ & $24.84(2.85)$ & $19.74(2.57)$ & 12.31 \\
\hline $\operatorname{HSR}(\mathrm{m} / \mathrm{min})$ & $5.15(3.48)$ & $5.59(4.01)$ & $1.02(1.68)$ & 64.30 \\
\hline VHIR (m/min) & $5.72(3.52)$ & 6.97 (5.33) & $1.02(1.68)$ & 68.66 \\
\hline PL (U.A./min) & $12.44(1.39)$ & $12.68(1.23)$ & $11.47(1.11)$ & 5.23 \\
\hline
\end{tabular}

HIR - high intensity running, MV - maximal velocity, HSR - high speed running, VHIR - very high intensity running, $\mathrm{PL}$ - player load, $S D$ - standard deviation, $C V$ - coefficient of variation of all sets 


\section{HUMAN MOVEMENT}

F. Clemente, A. Rabbani, R. Ferreira, J.P. Araújo, Performance dropping in small-sided games

Table 2. Between-set comparisons of external load variables reported as percentage and the probabilities of each standardized difference

\begin{tabular}{|c|c|c|c|c|}
\hline \multirow{2}{*}{ Format } & \multirow{2}{*}{ Variable } & \multicolumn{2}{|c|}{$\%$ difference (set 2 - set 1 ) } & \multirow{2}{*}{$\begin{array}{l}\% \text { greater/similar/lower values } \\
\text { for set } 2 \text { vs. set } 1\end{array}$} \\
\hline & & Value & $90 \% \mathrm{CI}$ & \\
\hline \multirow{8}{*}{ Set 1 vs. set 2} & Distance $(\mathrm{m} / \mathrm{min})$ & 3.0 & $-0.1,6.2$ & 68/32/1 Possible \\
\hline & $\operatorname{HIR}(\mathrm{m} / \mathrm{min})$ & 12.7 & $-12.8,45.6$ & 56/36/8 Unclear \\
\hline & $\mathrm{MV}(\mathrm{km} / \mathrm{h})$ & 1.5 & $-8.3,12.5$ & 45/29/26 Unclear \\
\hline & $\operatorname{HSR}(\mathrm{m} / \mathrm{min})$ & 11.1 & $-35.7,91.9$ & 40/42/18 Unclear \\
\hline & VHIR (m/min) & 17.1 & $-37.3,118.7$ & 47/37/17 Unclear \\
\hline & PL (U.A./min) & 2.1 & $-1.0,5.3$ & 40/58/1 Possible \\
\hline & & \multicolumn{2}{|c|}{$\%$ difference $(\operatorname{set} 3-\operatorname{set} 1)$} & $\%$ greater/similar/lower values \\
\hline & & Value & $90 \% \mathrm{CI}$ & for set 3 vs. set 1 \\
\hline \multirow{8}{*}{ Set 1 vs. set 3} & Distance (m/min) & -12.8 & $-17.3,-8.1$ & 0/0/100 Most likely \\
\hline & $\operatorname{HIR}(\mathrm{m} / \mathrm{min})$ & -62.6 & $-70.4,-52.7$ & 0/0/100 Most likely \\
\hline & $\mathrm{MV}(\mathrm{km} / \mathrm{h})$ & -19.4 & $-25.0,-13.5$ & 0/0/100 Most likely \\
\hline & $\operatorname{HSR}(\mathrm{m} / \mathrm{min})$ & -80.2 & $-95.6,-10.7$ & 3/2/94 Likely \\
\hline & VHIR (m/min) & -83.0 & $-96.4,-19.4$ & 3/2/95 Very likely \\
\hline & PL (U.A./min) & -7.6 & $-12.8,-2.2$ & 0/5/94 Likely \\
\hline & & \multicolumn{2}{|c|}{$\%$ difference (set 3 - set 2 ) } & $\%$ greater/similar/lower values \\
\hline & & Value & $90 \% \mathrm{CI}$ & for set 3 vs. set 2 \\
\hline \multirow{6}{*}{ Set 2 vs. set 3} & Distance $(\mathrm{m} / \mathrm{min})$ & -15.3 & $-18.6,-12.0$ & 0/0/100 Most likely \\
\hline & $\operatorname{HIR}(\mathrm{m} / \mathrm{min})$ & -66.8 & $-78.3,-49.3$ & 0/0/100 Most likely \\
\hline & $\mathrm{MV}(\mathrm{km} / \mathrm{h})$ & -20.7 & $-29.6,-10.6$ & 0/0/100 Most likely \\
\hline & $\operatorname{HSR}(\mathrm{m} / \mathrm{min})$ & -67.3 & $-94.2,84.5$ & 9/6/85 Unclear \\
\hline & VHIR (m/min) & -69.1 & $-94.5,74.4$ & 8/6/85 Unclear \\
\hline & PL (U.A./min) & -9.5 & $-13.2,-5.6$ & 0/0/100 Most likely \\
\hline
\end{tabular}

HIR - high intensity running, MV - maximal velocity, HSR - high speed running, VHIR - very high intensity running, PL - player load, CI - confidence interval

(64.30\%) and the VHIR (68.66\%) presented the highest coefficients of variation $(\mathrm{CV})$, and the distance covered (8.64\%) and PL (5.23\%) had the lowest variations.

Percentage, standardized differences, and probabilities of standardized differences of external load measures between sets can be found in Table 2 and Figures 1-3.

Possibly small increases of distance covered were found from set 1 to set 2 (3.0\% [-0.1, 6.2], ES: 0.27 $[-0.01,0.56])$. The remaining standardized differences between the first and the second sets were trivial.

Most likely very large decreases of HIR $(-62.6 \%$ [-70.4, -52.7], ES: -2.04 [-2.52, -1.55]) were found from set 1 to set 3 . Most likely large decreases of distance covered $(-12.8 \%[-17.3,-8.1], E S:-1.28$ [-1.77, $-0.79])$ and MV (-19.4\% [-25.0, -13.5], ES: -1.95 $[-2.60,-1.31])$ were observed from the first to the third set. Likely large decreases of HSR $(-80.2 \%$ [-95.6, -10.7], ES: -1.78 [-3.43, -0.12]), moderate decreases

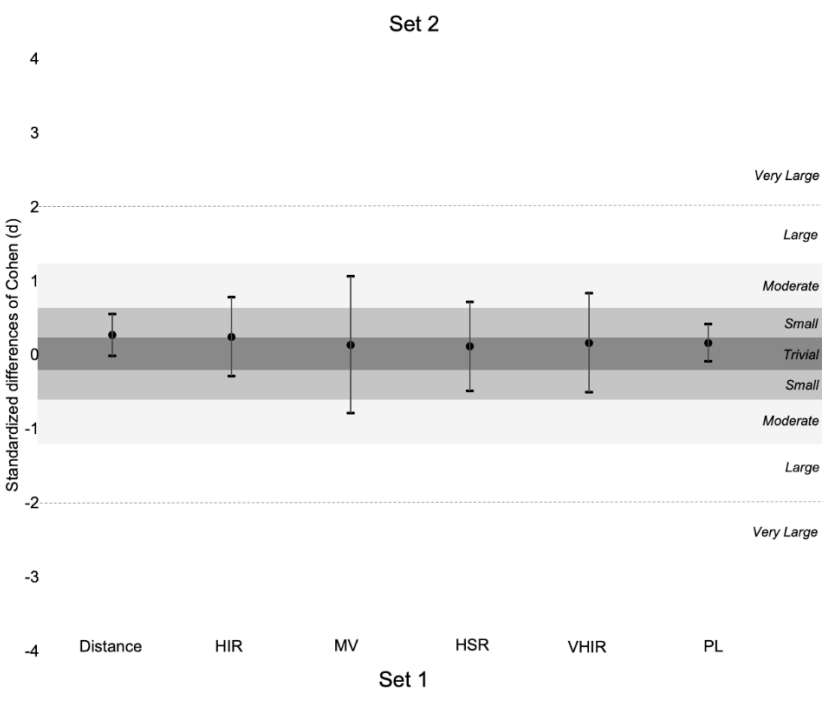

HIR - high intensity running, MV - maximal velocity, HSR - high speed running, VHIR - very high intensity running, PL - player load

Figure 1. Standardized differences of Cohen $(d)$ between the first and the second sets for the external load variables 


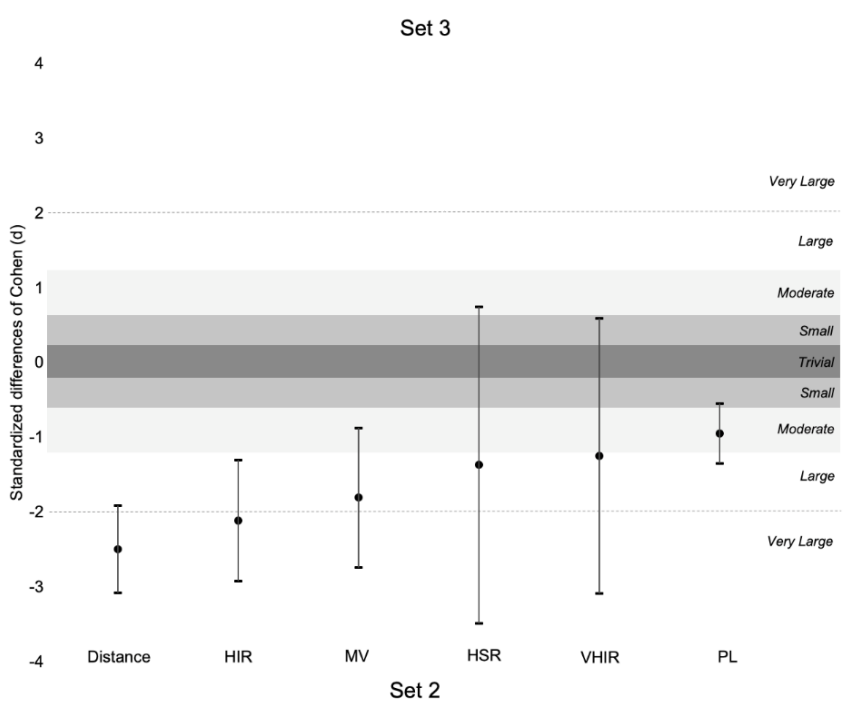

HIR - high intensity running, MV - maximal velocity, HSR - high speed running, VHIR - very high intensity running, $\mathrm{PL}$ - player load

Figure 3. Standardized differences of Cohen $(d)$ between the second and third sets for the external load variables

up effect) [22]. No significant differences between sets were reported while comparing heart rate responses between 3 sets of 2, 4, and 6 minutes during the 3 vs. 3 format [15]. Opposite to these findings, a study performed over 4 sets of 4 minutes with a 4 vs. 4 format revealed that the last set significantly increased the players' heart rate responses and perceived effort [23]. Overall, the findings regarding the variations in acute physiological responses across different sets during SSGs do not seem to present major differences. However, the physical demands imposed during the match can be highly variable, independently of heart rate variations, mainly considering a previous study that reported that a continuous SSG regimen induced greater physical loads than intermittent regimens [24]. From that perspective, our study analysed variations in external load measures (objectively determined by a GPS system) during 3 sets of a conditioned SSG format played by professional players.

The results obtained in the present study suggest that there is a clear dropping tendency in players' capacities to sustain the initial standards of physical participation during SSGs. It was found that the third set promoted very large drops in HIR $(-62.6 \%$ and $-66.8 \%$ when compared with the first and the second sets, respectively) and total distance $(-15.3 \%$ when compared with the second set). These drops are in line with a previous study that reported a progressive and significant decremental effect of sets on distance and HIR during 4 sets of 4 minutes played in a 4 vs. 4 format [23]. This might be explained by the progres- 
F. Clemente, A. Rabbani, R. Ferreira, J.P. Araújo, Performance dropping in small-sided games

sive accumulation of blood lactate concentrations across sets as revealed in a study conducted with different SSG formats [22]. Moreover, the resting period between sets may not allow players to sustain high enough levels of deoxygenated haemoglobin to avoid the decremental effect of progressive exercises during SSGs [25].

The third set also promoted large decreases in HSR (-80.2 and $-67.3 \%$ when compared with the first and second sets, respectively) and VHIR (-83.0 and $-69.1 \%$ when compared with the first and second sets, respectively). In the above-mentioned study conducted with a 4 vs. 4 format [23], a progressive and significant decrease in sprinting was also recorded.

However, the results of our study are particularly impressive if one considers the magnitudes of changes and their implications for sports training. In fact, the high increment of $C V$ (relative $S D$ ) within sets for these 2 measures must also be taken into account. In the first and the second sets, the CVs of $67.6 \%$ and $71.7 \%$, respectively, can be observed in HSR. However, an increase of $164.7 \% \mathrm{CV}$ was obtained during the third and last sets. Also, in the case of VHIR, CVs of $61.5 \%$ and $76.5 \%$ were noted in the first and second sets, respectively, and an increment of $165 \%$ in the third set. These great increments of variability within sets may represent a major consequence of training and may indicate the unpredictability of the proper stimulation of these 2 important measures in players.

In fact, the decrease and increase in variability within sets may not be a direct consequence of accumulated fatigue. A study that tested the effects of 2 recovery periods (30 seconds vs. 120 seconds) during SSGs revealed that the 30 -second rest period resulted in a significant increase in deoxygenated haemoglobin. However, this increase did not alter the physiological or physical performance of players during the games [25]. The authors explained such results by citing the possible self-regulation of players during the task [25]. Following such an explanation, another study tested the effects of different set durations in SSGs and verified that players adjusted their pacing strategies on the basis of the time of exercise [26]. In fact, earlier knowledge of the exercise duration seems to contribute to increases in the intensity and effort exerted by players during short sets and decreases in the intensity of physical demands during longer ones [27]. Consequently, it seems that a player's experience may compromise their effort during SSGs, particularly if the main goal is to develop a stimulus that meets the adequate percentage of maximal aerobic capacity. Thus, the intensity in SSGs may vary depending on many concurrent fac- tors that should be considered during the monitoring process and the implementation of these games [24].

This study had some limitations. Acute physiological stimuli (e.g., heart rate, perceived effort, and blood lactate concentrations) were not measured and possibly would be important to the association between drops in the levels of physical demands and changes in internal load. Moreover, the small sample size of the present study does not allow the findings to be generalized. The fact of establishing one single session may also be considered as a limitation and therefore future studies should include multiple sessions to confirm the evidence found in our study. Another limitation was not relating the fitness status of the players with the external performance during SSGs and the drops associated. Finally, additional tactical analysis should be considered to relate the player's behaviour with the variations in physical level during these games [28].

Despite these limitations, the findings presented in this study suggest that coaches should be aware of the physical decrements throughout SSGs sets. A major drop in physical demands should be carefully analysed in the context of the coach's goals. It might be necessary to increase resting periods, to adjust the amount of verbal encouragement given, or even to change the structure/goals of the game in order to deregulate the self-pacing strategies of players and increase the demands of the task.

\section{Conclusions}

The study revealed that the third set of 4 minutes during a GK +5 vs. 5 + GK conditioned SSG format significantly contributed to large decreases in the physical demands of professional soccer players. Moreover, increases in the variability of HSR and VHIR within the exercise were observed in the last set. These results should be carefully interpreted by coaches, especially if the aim of training tasks is to develop the players' fitness levels. The self-regulation pacing strategies of experienced and professional players may lead to homogeneity in the physical stimuli across sets. For that reason, coaches should use complementary strategies to avoid declines in external load.

\section{Disclosure statement}

No author has any financial interest or received any financial benefit from this research.

\section{Conflict of interest}

The authors state no conflict of interest. 


\section{References}

1. Halouani J, Chtourou H, Gabbett T, Chaouachi A, Chamari K. Small-sided games in team sports training: a brief review. J Strength Cond Res. 2014;28(12):35943618; doi: 10.1519/JSC.0000000000000564.

2. Clemente FM, Nikolaidis PT, Van Der Linden CMIN, Silva B. Effects of small-sided soccer games on internal and external load and lower limb power: a pilot study in collegiate players. Hum Mov. 2017;18(1):50-57; doi: 10.1515/humo-2017-0007.

3. Owen AL, Wong DP, Paul D, Dellal A. Physical and technical comparisons between various-sided games within professional soccer. Int J Sports Med. 2014; 35(4):286-292; doi: 10.1055/s-0033-1351333.

4. Brandes M, Heitmann A, Müller L. Physical responses of different small-sided game formats in elite youth soccer players. J Strength Cond Res. 2012;26(5):13531360; doi: 10.1519/JSC.0b013e318231ab99.

5. Köklü Y. A comparison of physiological responses to various intermittent and continuous small-sided games in young soccer players. J Hum Kinet. 2012;31:89-96; doi: 10.2478/v10078-012-0009-5.

6. Halouani J, Chtourou H, Dellal A, Chaouachi A, Chamari K. Soccer small-sided games in young players: rule modification to induce higher physiological responses. Biol Sport. 2017;34(2):163-168; doi: 10.5114/ biolsport.2017.64590.

7. Lacome M, Simpson BM, Cholley Y, Lambert P, Buchheit M. Small-sided games in elite soccer: does one size fit all? Int J Sports Physiol Perform. 2018;13(5):568576; doi: 10.1123/ijspp.2017-0214.

8. Rampinini E, Impellizzeri F, Castagna C, Abt G, Chamari K, Sassi A, et al. Factors influencing physiological responses to small-sided soccer games. J Sports Sci. 2007;25(6):659-666;doi:10.1080/02640410600811858.

9. Katis A, Kellis E. Effects of small-sided games on physical conditioning and performance in young soccer players. J Sports Sci Med. 2009;8(3):374-380.

10. Clemente FM, Lourenço Martins FM, Mendes RS. Developing aerobic and anaerobic fitness using small-sided soccer games: methodological proposals. Strength Cond J. 2014;36(3):76-87; doi: 10.1519/SSC.00000000000 00063.

11. Sarmento H, Clemente FM, Harper LD, da Costa IT, Owen A, Figueiredo AJ. Small sided games in soccer - a systematic review. Int J Perform Anal Sport. 2018;18(5) :693-749; doi: 10.1080/24748668.2018.1517288.

12. Radziminski L, Rompa P, Barnat W, Dargiewicz R, Jastrzebski Z. A comparison of the physiological and technical effects of high-intensity running and smallsided games in young soccer players. Int J Sports Sci Coach. 2013;8(3):455-465; doi: 10.1260/1747-9541. 8.3.455.

13. Hourcade J-C, Noirez P, Sidney M, Toussaint J-F, Desgorces F-D. Performance losses following threefold volume increases in soccer-specific training and in small-sided games. Sci Med Footb. 2019;3(1):3-13; doi: 10.1080/24733938.2018.1486038.

14. Sparkes W, Turner A, Weston M, Russell M, Johnston M, Kilduff L. Neuromuscular, biochemical, endocrine, and mood responses to small-sided games' training in professional soccer. J Strength Cond Res. 2018;32(9):25692576; doi: 10.1519/JSC.0000000000002424.

15. Fanchini M, Azzalin A, Castagna C, Schena F, McCall A, Impellizzeri FM. Effect of bout duration on exercise intensity and technical performance of small-sided games in soccer. J Strength Cond Res. 2011;25(2):453-458; doi: 10.1519/JSC.0b013e3181c1f8a2.

16. Köklü Y, Alemdaroğlu U, Cihan H, Wong DP. Effects of bout duration on players' internal and external loads during small-sided games in young soccer players. Int J Sports Physiol Perform. 2017;12(10):1370-1377; doi: 10.1123/ijspp.2016-0584.

17. Hill-Haas SV, Dawson B, Impellizzeri FM, Coutts AJ. Physiology of small-sided games training in football: a systematic review. Sports Med. 2011;41(3):199-220; doi: 10.2165/11539740-000000000-00000.

18. Johnston RJ, Watsford ML, Kelly SJ, Pine MJ, Spurrs RW. Validity and interunit reliability of $10 \mathrm{~Hz}$ and $15 \mathrm{~Hz}$ GPS units for assessing athlete movement demands. J Strength Cond Res. 2014;28(6):1649-1655; doi: 10.1519/ JSC.0000000000000323.

19. Cohen J. Statistical power analysis for the behavioral sciences. New York: Lawrence Erlbaum Associates; 1988.

20. Batterham AM, Hopkins WG. Making meaningful inferences about magnitudes. Int J Sports Physiol Perform. 2006;1(1):50-57; doi: 10.1123/ijspp.1.1.50.

21. Hopkins WG, Marshall SW, Batterham AM, Hanin J. Progressive statistics for studies in sports medicine and exercise science. Med Sci Sports Exerc. 2009;41(1):313; doi: 10.1249/MSS.0b013e31818cb278.

22. Köklü Y, Aşçi A, Koçak FÜ, Alemdaroğlu U, Dündar U. Comparison of the physiological responses to different small-sided games in elite young soccer players. J Strength Cond Res. 2011;25(6):1522-1528; doi: 10.1519/JSC. 0b013e3181e06ee1.

23. Dellal A, Lago-Penas C, Wong del P, Chamari K. Effect of the number of ball contacts within bouts of 4 vs. 4 small-sided soccer games. Int J Sports Physiol Perform. 2011;6(3):322-333; doi: 10.1123/ijspp.6.3.322.

24. Casamichana D, Castellano J, Dellal A. Influence of different training regimes on physical and physiological demands during small-sided soccer games: continuous vs. intermittent format. J Strength Cond Res. 2013; 27(3):690-697; doi: 10.1519/JSC.0b013e31825d99dc.

25. McLean S, Kerhervé H, Lovell GP, Gorman AD, Solomon $\mathrm{C}$. The effect of recovery duration on vastus lateralis oxygenation, heart rate, perceived exertion and time motion descriptors during small sided football games. PLoS One. 2016;11(2):e0150201; doi: 10.1371/ journal.pone.0150201. 


\section{HUMAN MOVEMENT}

F. Clemente, A. Rabbani, R. Ferreira, J.P. Araújo, Performance dropping in small-sided games

26. Sampson JA, Fullagar HH, Gabbett T. Knowledge of bout duration influences pacing strategies during smallsided games. J Sports Sci. 2015;33(1):85-98; doi: 10.1080/02640414.2014.925571.

27. Ferraz R, Gonçalves B, Van Den Tillaar R, Jiménez Sáiz S, Sampaio J, Marques MC. Effects of knowing the task duration on players' pacing patterns during soccer small-sided games. J Sports Sci. 2018;36(1): 116-122; doi: 10.1080/24733938.2017.1283433.

28. Sarmento H, Clemente FM, Araújo D, Davids K, McRobert A, Figueiredo A. What performance analysts need to know about research trends in association football (2012-2016): a systematic review. Sports Med. 2018; 48(4):799-836; doi: 10.1007/s40279-017-0836-6. 